\title{
Labor de proyeccion humanitaria de la FlLACP. Campaña 2019
}

\section{FlLACP humanitarian projection work. Gampaign 2019}

\author{
Teresa ZAMBRANA ROJAS* ${ }^{*}$ Alfonso VALLARTA RODRÍGUEZ** \\ Alejandro DUARTE y SÁNCHEZ***
}

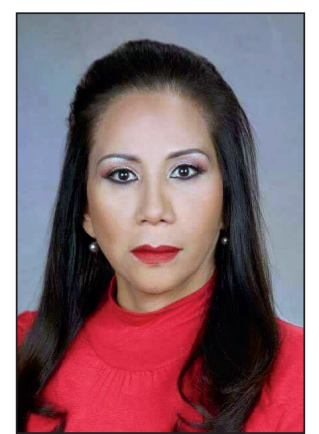

Zambrana Rojas T.

Resumen

Introducción y objetivo. La Federación Ibero Latinoamericana de Cirugía Plástica (FILACP), como organización internacional de especialistas en Cirugía Plástica, Estética y Reparadora unidos por una lengua común, incentiva la participación de sus cirujanos en campañas de labor humanitaria que, prestando ayuda médica con cirugías de alta complejidad a pacientes de entornos sociales o geográficos menos favorecidos, pueden mejorar su calidad de vida y su integración en la sociedad.

Presentamos en este trabajo, a modo de ejemplo de una labor iniciada ya hace años, la desarrollada por los equipos que han participado en la III Jornada Altruista de Cirugía Plástica de la FILACP correspondiente al año 2019.

Material y método. La campaña fue convocada desde FILACP a través de su página web, la página de Facebook y el canal de Youtube en redes sociales y mediante cartas enviadas a los delegados de sus Comités Médico Social y de Difusión y a los presidentes de las sociedades nacionales de Cirugía Plástica de los 22 países miembros.

Las jornadas de trabajo se desarrollaron durante los meses de julio y agosto de 2019 con equipos quirúrgicos compuestos por cirujanos plásticos, anestesiólogos y personal de enfermería que trabajaron voluntariamente, de forma altruista, y tanto a título personal como apoyados por las instituciones sanitarias en las que habitualmente trabajan o por fundaciones con las que colaboran.

Resultados. En total fueron intervenidos quirúrgicamente 1008 pacientes ( $78.75 \%$ de los vistos en consulta), con una media de edad de 6.9 años, $51 \%$ varones y $49 \%$ mujeres. Los 3 grupos principales de patologías atendidas fueron: fisuras labiopalatinas $(31 \%)$, reconstrucción mamaria postmastectomía $(24 \%)$ y quemaduras $(17 \%)$.

Conclusiones. A través de este trabajo La FILACP agradece a todos y cada uno de los cirujanos que participaron en esta III Jornada Altruista y reconoce a los que lo hicieron en campañas anteriores, da a conocer datos de la actividad quirúrgica realizada en esta ocasión y contribuye a difundir la vertiente reconstructiva de la especialidad y su importante contribución a la mejora de la calidad de vida de los pacientes más desfavorecidos.

Palabras clave Cirugía Plástica humanitaria, Labio paladar hendido, Reconstrucción mamaria, Quemaduras

Nivel de evidencia científica Recibido [esta versión] Aceptado
5 Terapéutico

30 septiembre/2019

30 noviembre/2019
Background and objective. The Ibero Latinamerican Federation of Plastic Surgery (FILACP), as an international organization of Plastic, Aesthetic and Reconstructive Surgery specialists with a common language, encourages their collaboration in humanitarian campaigns where with their work in highly complex surgeries on patients in disadvantaged environments, try to improve their quality of life and their social integration.

In this paper we present, as an example of the last years work, the activities developed by the teams enrolled in the III Plastic Surgery Altruist Campaign of FILACP in 2019

Methods. This campaign was convened from FILACP web, Fecebook and Youtube social networks and through letters sent to delegates in Social Medical and Communications Committees and to the presidents of national societies in the 22 member countries.

The surgical working days were developed during July and August with teams composed by plastic surgeons, anesthesiologists and nursing staff who worked voluntarily, altruistically, and both in personal capacity or supported by the health institutions in which they usually work or by foundations with which they collaborate.

Results. A total of 1008 patients were operated $(78.75 \%$ of those visited in the consultation), with an average age of 6.9 years, $51 \%$ men and $49 \%$ women. The 3 main groups of attended pathologies were: lip and palate cleft (31\%), post mastectomy breast reconstruction (24\%) and burns $(17 \%)$.

Conclusions. With this paper FILACP wants to thank each and every surgeon who participated in this III Altruist Campaign and recognize those who did it in previous campaigns, presents data from surgical activity conducted this time and contributes to the knowledge of the reconstructive aspect of the specialty and its important contribution to the improvement of the quality of life of the most disadvantaged patients.

Key worls Humanitarian Plastic Surgery, Lip and palate clefts, Breast reconstruction, Burns sequels

\section{Level of evidence Received [this version] Accepted}

5 Therapeutic
30 September/2019 30 November $/ 2019$

Conflicto de intereses: Los autores declaran no tener ningún interés financiero relacionado con el contenido de este artículo. Financiación: No hubo fuentes externas de financiación para este trabajo. 


\section{Introducción}

La Federación Ibero Latinoamericana de Cirugía Plástica (FILACP) es una entidad internacional fundada en el año 1974 en Caracas (Venezuela) que agrupa a las Sociedades Nacionales de Cirugía Plástica de los 22 países Latinoamericanos y de la Península Ibérica unidos por dos lenguas comunes, el español y el portugués. En la actualidad cuenta con más de 6.000 cirujanos plásticos que conforman una organización mundialmente reconocida y con una creciente actividad científica e institucional. ${ }^{(1)}$

Entre sus objetivos fundacionales se encuentran el asistir en la formación de sociedades nacionales de la especialidad; celebrar congresos internacionales cada 2 años; promover publicaciones científicas a través de su órgano oficial, la revista Cirugía Plástica Ibero-Latinoamericana; cuidar la ética de sus miembros; promover la preparación de postgrado en Cirugía Plástica e instituir premios y becas a través de su Fundación Docente.

Una parte importante de la labor del médico, de su compromiso deontológico y de su responsabilidad con la sociedad que le rodea, es realizar también un trabajo altruista hacia la población más desfavorecida, aportando con sus conocimientos y destrezas la solución de diferentes dolencias. Nuestra especialidad, la Cirugía Plástica, Estética y Reparadora, capaz de trabajar en toda la anatomía corporal manejando aspectos oncológicos, reconstructivos, malformaciones congénitas, quemaduras, reimplantes, etc., es capaz de hacer una gran aportación en este aspecto social, y esto se demuestra en la habitual colaboración anónima de los especialistas iberolatinoamericanos con fundaciones, organizaciones gubernamentales y no gubernamentales, entidades privadas, religiosas o militares. Esta labor se suele llevar a cabo en entornos sanitarios en desarrollo, con pacientes que no pueden acceder a una atención especializada por encontrarse en ambientes pobres, desfavorecidos socialmente o en los que por razones geográficas o políticas no pueden recibir una atención sanitaria especializada, y ha sido publicada en numerosas artículos en las páginas de esta misma revista, Cirugía Plástica Ibero-Latinoamericana, artículos que están disponibles en acceso abierto desde la propia página web de la revista. ${ }^{(2)}$

Consciente de todo lo que en este terreno de colaboración altruista se puede hacer en el ámbito de los países latinoamericanos de la FILACP, esta entidad promueve en los últimos años Campañas Humanitarias de Cirugía Reconstructiva en las que llama a la participación desinteresada de sus miembros en sus respectivos países, la mayoría de las veces en colaboración con sus sociedades nacionales de Cirugía Plástica y con las instituciones sa- nitarias o fundaciones en las que trabajan o participan. Esta labor se coordina desde la Presidencia de la FILACP, a través de su Director de Relaciones Internacionales y en colaboración con el Comité Médico-Social.

En este trabajo presentamos la actividad desarrollada durante la campaña convocada en el año 2019, como ejemplo del desarrollo que se ha ido alcanzando con el tiempo en la organización de esta compleja labor que implica a tantos profesionales y en tantos países de manera simultánea, y como constancia en datos del volumen de pacientes que se benefician de ella.

\section{Material y método}

En 2019, la FILACP, lanzó una convocatoria para su III Jornada Altruista de Cirugía Reconstructiva a celebrar durante los meses de julio y agosto en los países de su entorno. La difusión se hizo a través de anuncios colocados en la web institucional www.filacp.org, en su página de Facebook y en su canal de Youtube en redes sociales y mediante carta enviada a los delegados nacionales del Comité Médico Social y del Comité de Difusión en los 22 países miembros. Esta carta fue firmada por el Presidente de FILACP, Dr. Alejandro Duarte y la coordinación de la campaña corrió a cargo de la Dirección de Relaciones Internacionales, con el Dr. Alfonso Vallarta al frente, y el Comité Médico-Social, dirigido por la Dra. Teresa Zambrana.

Las actividades quirúrgicas a desarrollar incluirían: cirugías de labio y paladar hendidos; secuelas de quemaduras; traumatismos de mano y extremidades; patologías congénitas de todo tipo; y reconstrucción mamaria postcáncer.

La finalidad de la campaña, al igual que en sus convocatorias anteriores, sería ayudar a la población más desfavorecida en los distintos entornos nacionales y contribuir a modificar la principal imagen que desde la población en general se tiene de los cirujanos plásticos, con un enfoque principalmente hacia la Cirugía Estética que olvida la importante vertiente de la Cirugía Reconstructiva que estos especialistas también desarrollan como parte fundamental de su especialidad y que tanto bien proporciona a la sociedad.

La respuesta desde la mayoría de los países de la FILACP fue inmediata y se crearon grupos de servicio altruista con equipos quirúrgicos que en unas ocasiones estuvieron integrados por especialistas del mismo entorno laboral, apoyados por su institución, y en otras por grupos nacionales en los países más pequeños; algunos incluso contaron con apoyo gubernamental. En todos los casos se constituyeron grupos multidisciplinarios integrados por cirujanos plásticos, anestesiólogos y personal 
de enfermería, todos ellos adecuadamente cualificados, que otorgaron de manera organizada sus servicios con gran gusto y satisfacción.

Los interesados en participar comunicaron con la dirección ofrecida desde FILACP para la coordinación general de los grupos y recuento de actividades a través de la dirección medicosfilacpsocial@gmail.com; fueron integrados en el grupo final de actividades y se les envió plantilla para recogida de datos.

\section{Resultados}

En la campaña de 2019 participaron cirujanos plásticos de 11 países: Argentina, Brasil, Bolivia, Colombia, Chile, Cuba, Ecuador, México, Nicaragua, Paraguay y Perú (Fig. 1-12).

En total, fueron vistos en consulta 1280 pacientes de los que finalmente fueron sometidos a tratamiento quirúrgico 1008 (78.75\%) (Gráfico 1). Por sexos: 515 pacientes fueron mujeres $(51.08 \%)$ y 493 varones $(49.89 \%)$. El rango de edad estuvo entre los 2 meses y los 76 años, con una media de 9.6 años (Tabla I, Gráfico 2).

Las patologías intervenidas fueron: 313 pacientes fisurados (31\%), 172 con secuelas de quemaduras (17\%), 242 reconstrucciones mamarias postmastectomía por cáncer (24\%) y 281 pacientes en el grupo de miscelánea (28\%) (Tabla II, Gráfico 3).

Gráfico 1. Distribución de pacientes y de países participantes en la campaña

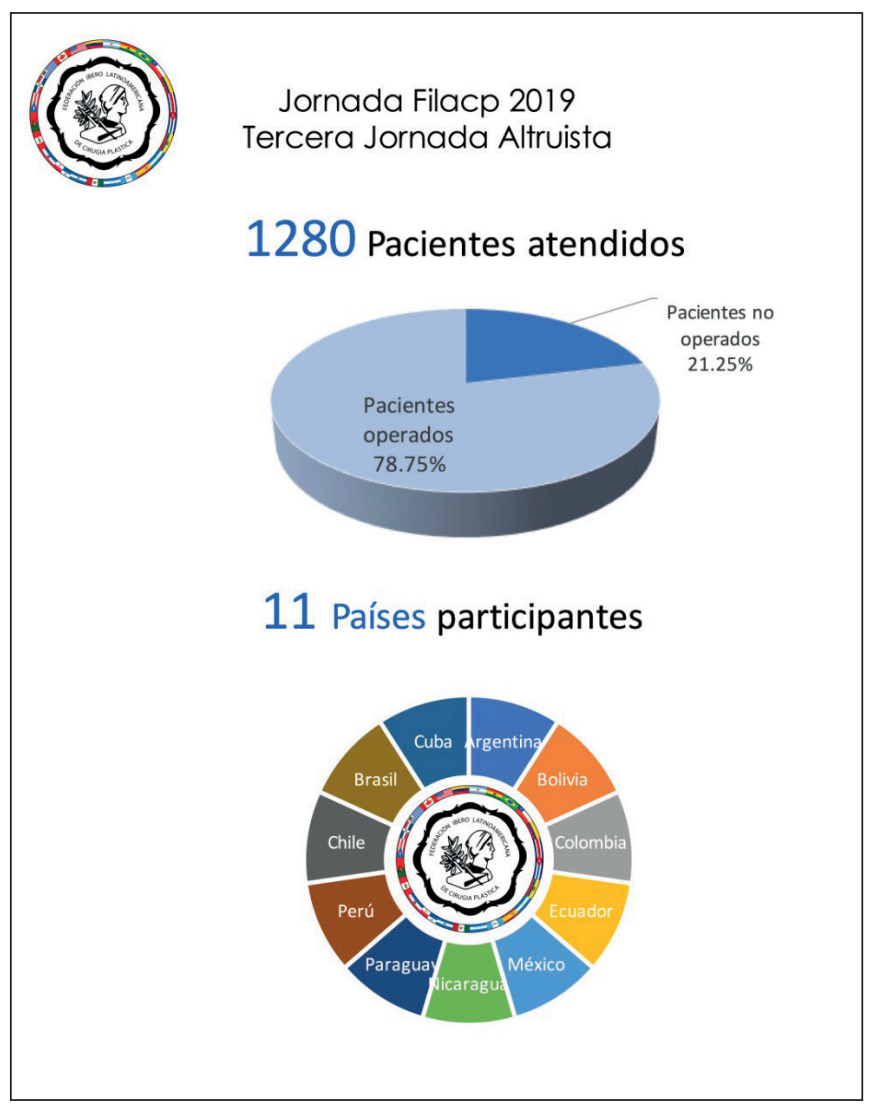

Tabla I. Datos demográficos del grupo de paciente intervenidos

\begin{tabular}{|ccc|}
\hline Total de pacientes & Varones & Mujeres \\
\hline 1008 & $493(49.89 \%)$ & $515(51.08 \%)$ \\
\hline
\end{tabular}

Tabla II. Intervenciones realizadas

\begin{tabular}{|lcc|}
\hline Patología & N $^{0}$ de pacientes & $\%$ \\
\hline Fisurados & 313 & 31 \\
Quemados & 172 & 17 \\
Rec. mama & 242 & 24 \\
Miscelánea & 281 & 28 \\
\hline
\end{tabular}

Gráfico 2. Distribución de los pacientes por sexo y edad

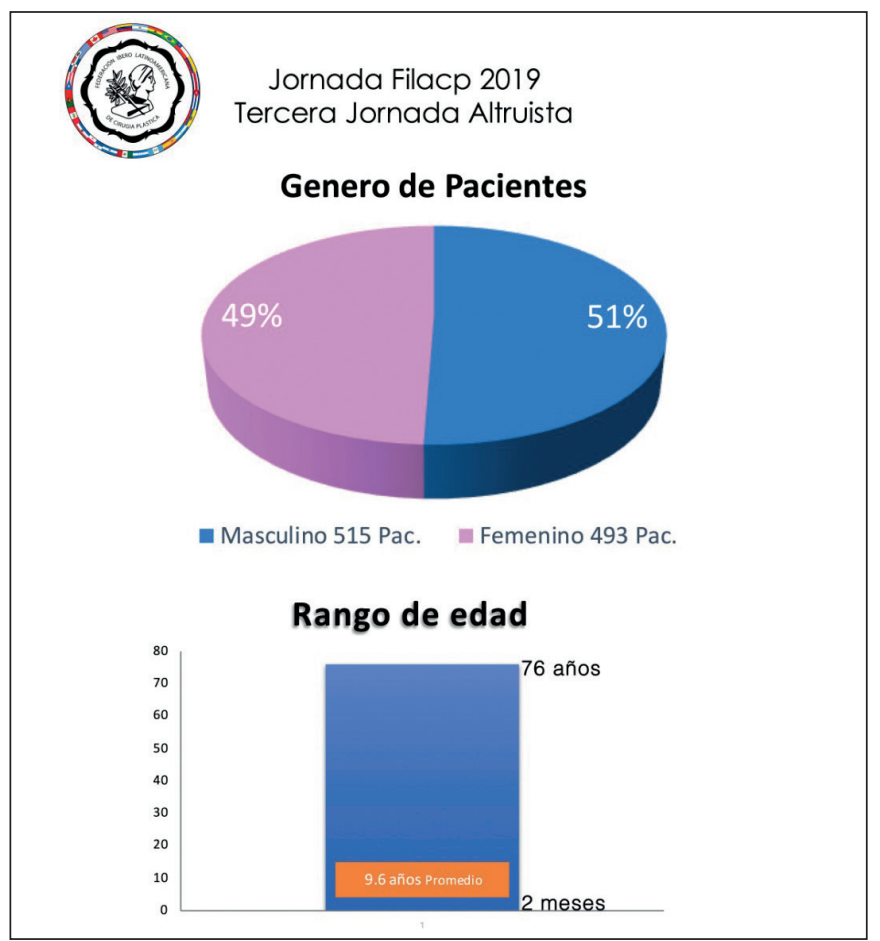

Gráfico 3. Distribución de los pacientes según el tipo de patología tratada

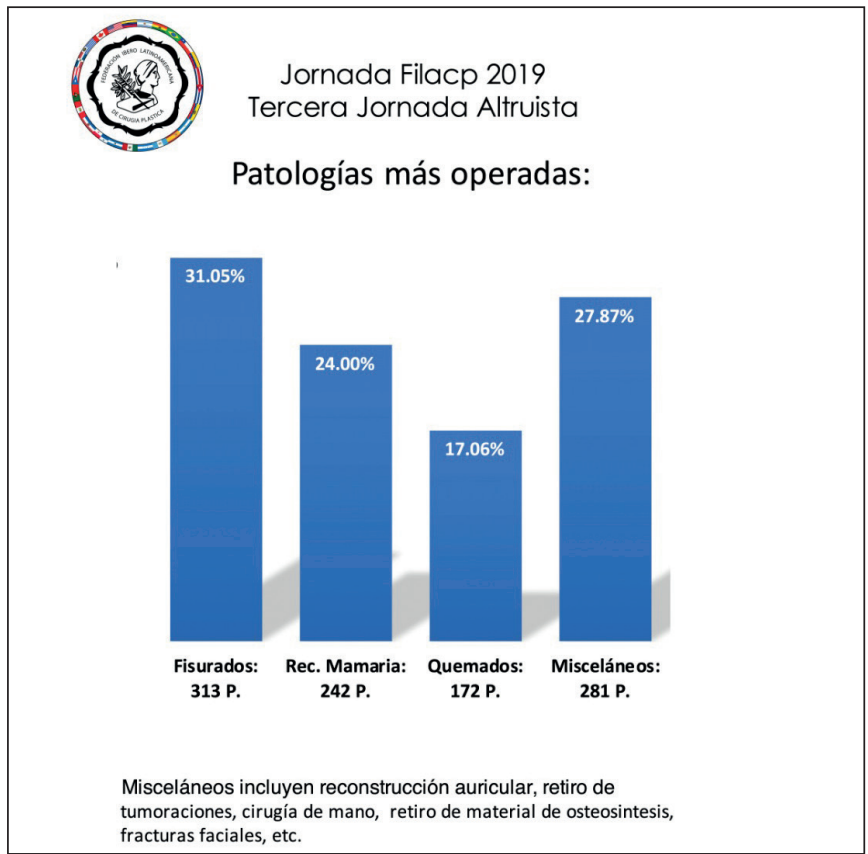




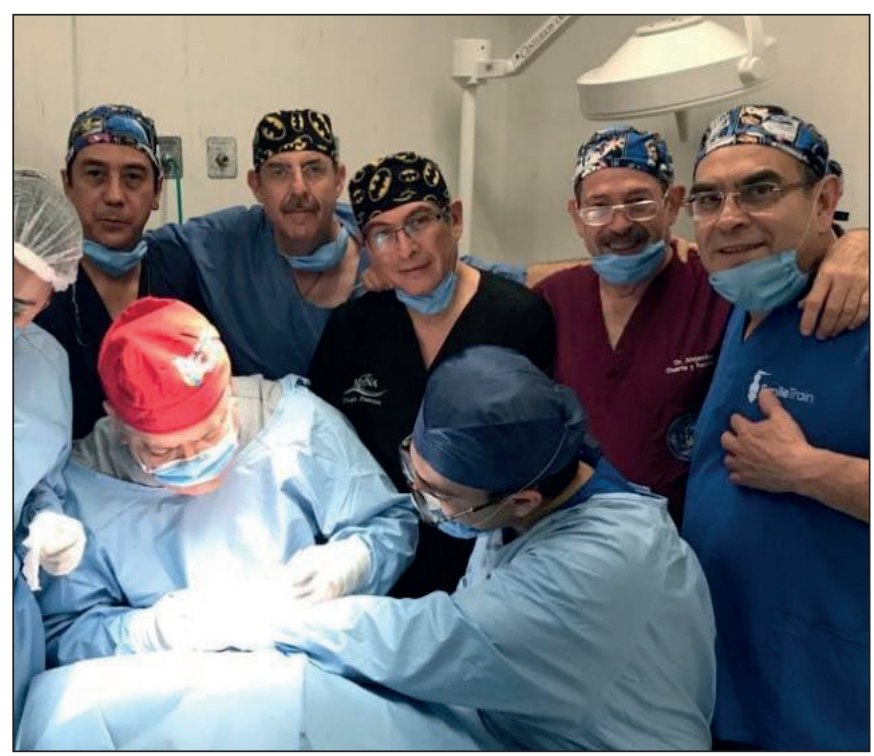

Figura 1. Equipo de los Dres. José Antonio Leon, Alfonso Miranda, José Mena, Alejandro Duarte, Blas Domínguez, Héctor Lino, en Ciudad de México

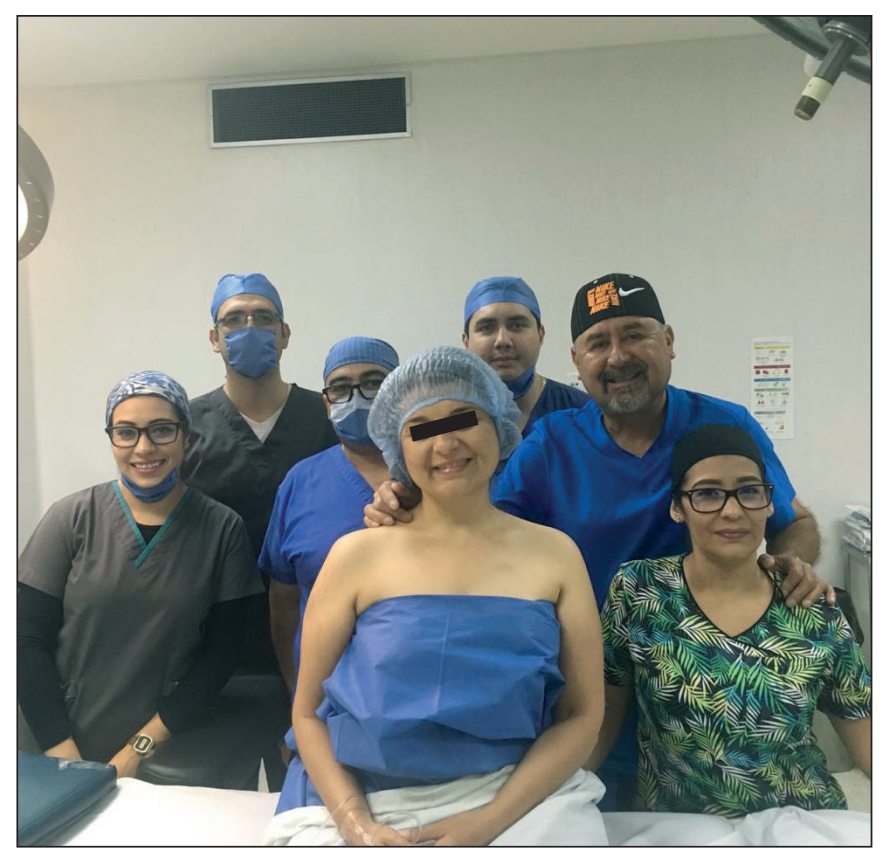

Figura 3. Equipo del Dr. Alfredo Carballo en La Paz, Baja California Sur, México.

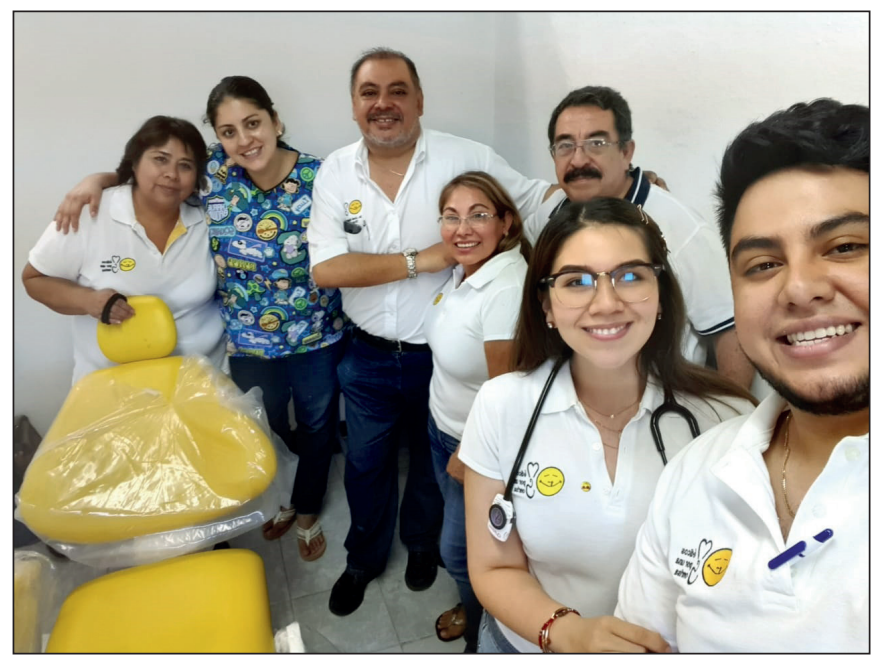

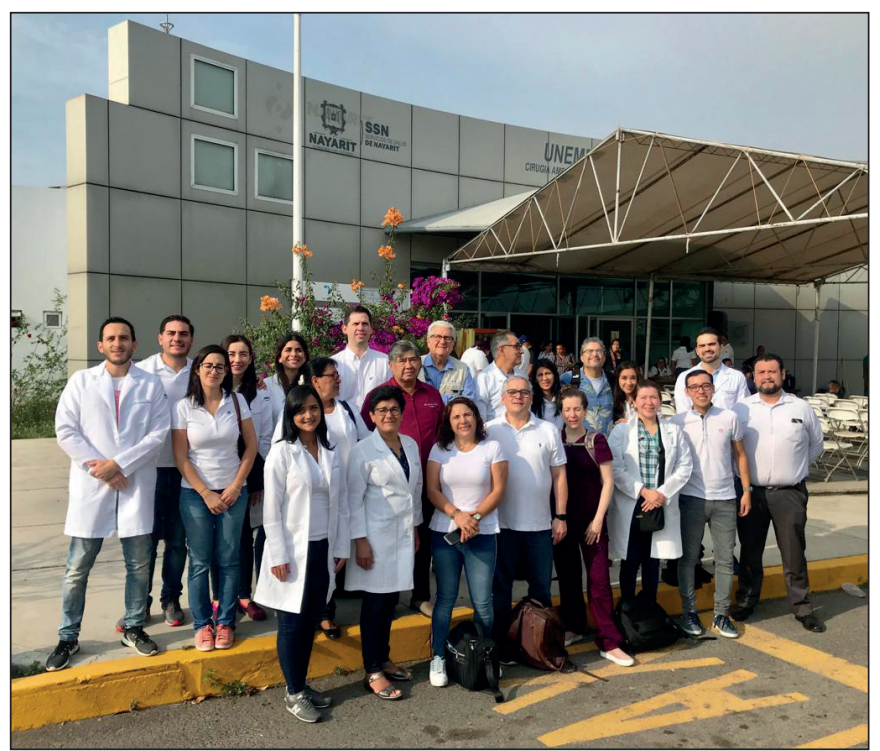

Figura 2. Equipo de los Dres. Miguel Viera, Ignacio Lugo, Martín Morales, David Trejo, Cynthia Euán, Arnoldo Topete, Jesús Escriva, Guillermo Castorena, Mariana Calderón y Alfonso Vallarta en Tepic, Nayarit, México.

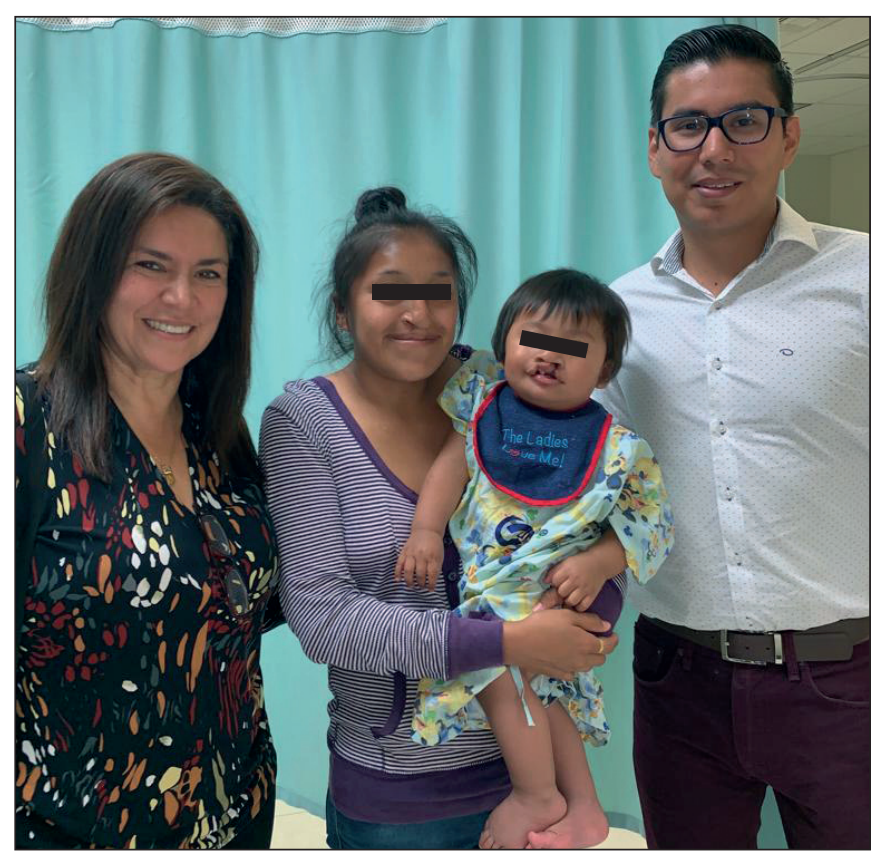

Figura 4. Equipo de la Dra. Alicia Sigler, en Ensenada, Baja California, México.

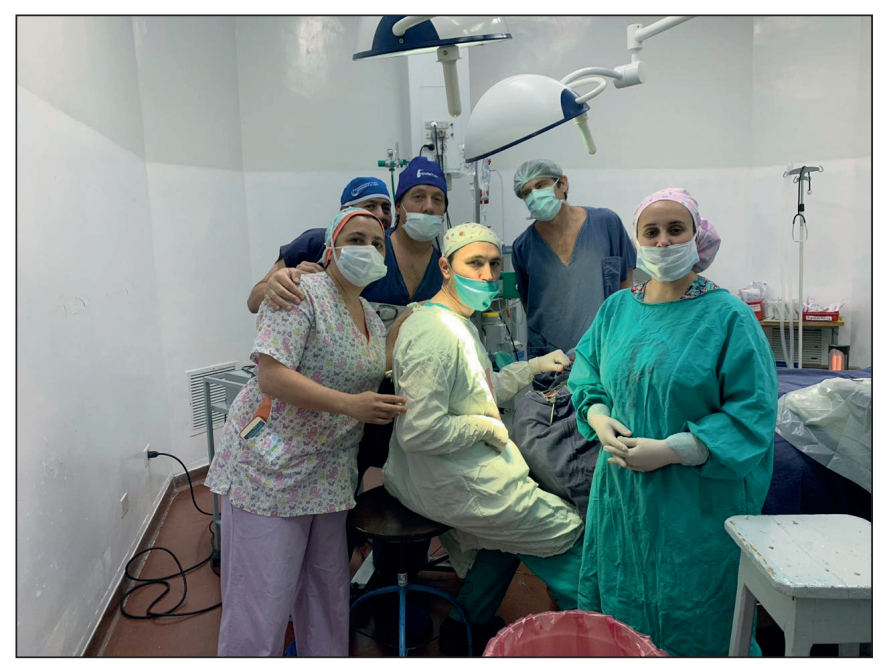

Figura 6. Equipo del Dr. Hugo Barbosa, Argentina. 


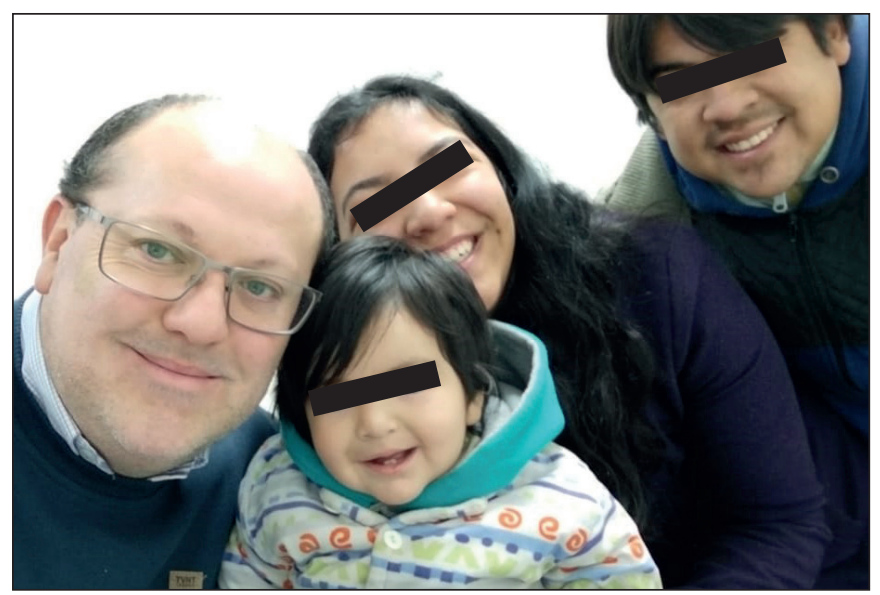

Figura 7. Dr. José Herboso, Argentina.

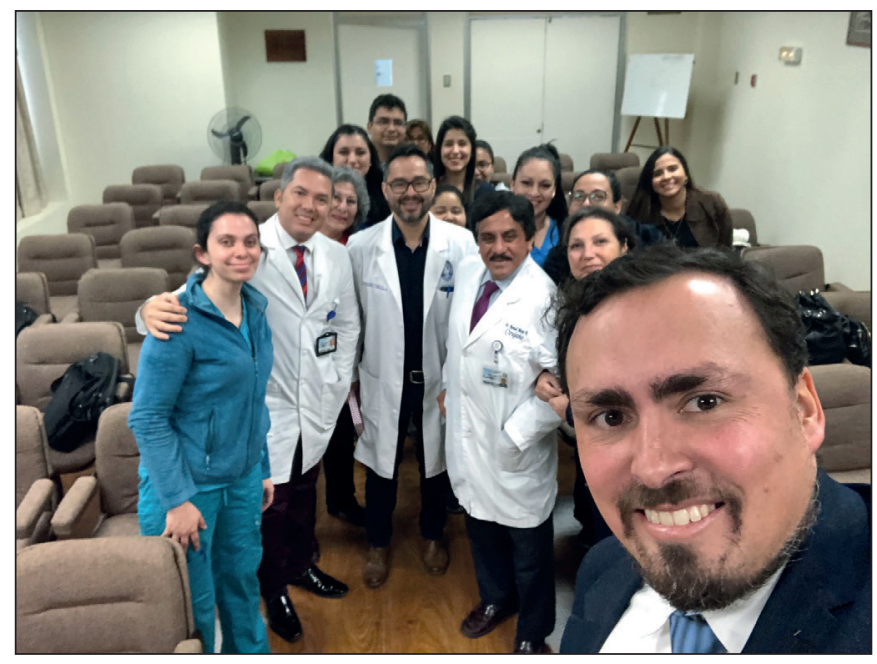

Figura 9. Equipo del Dr. Cristian Erazo, Chile.

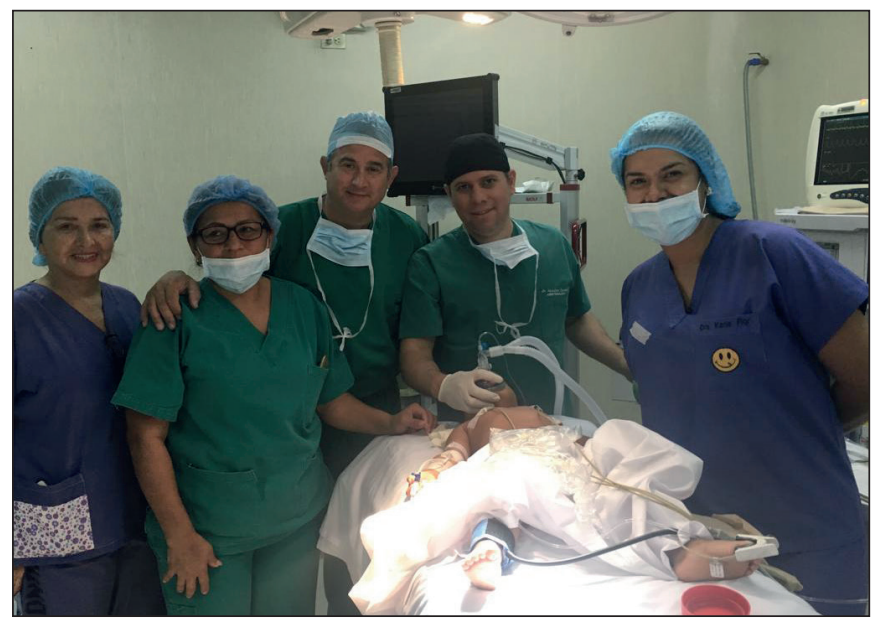

Figura 11. Equipo del Dr. Fernando Quintana, Ecuador.

Discusión

Como cirujanos plásticos, tenemos la gran dicha de que nuestra especialidad puede mejorar la calidad de vida de muchos pacientes utilizando nuestras habilidades y conocimientos para empezar con el diagnóstico y continuar con los procedimientos quirúrgicos necesarios, gracias a los que pacientes con malformaciones, secuelas que comprometen su función y su calidad de vida, y por

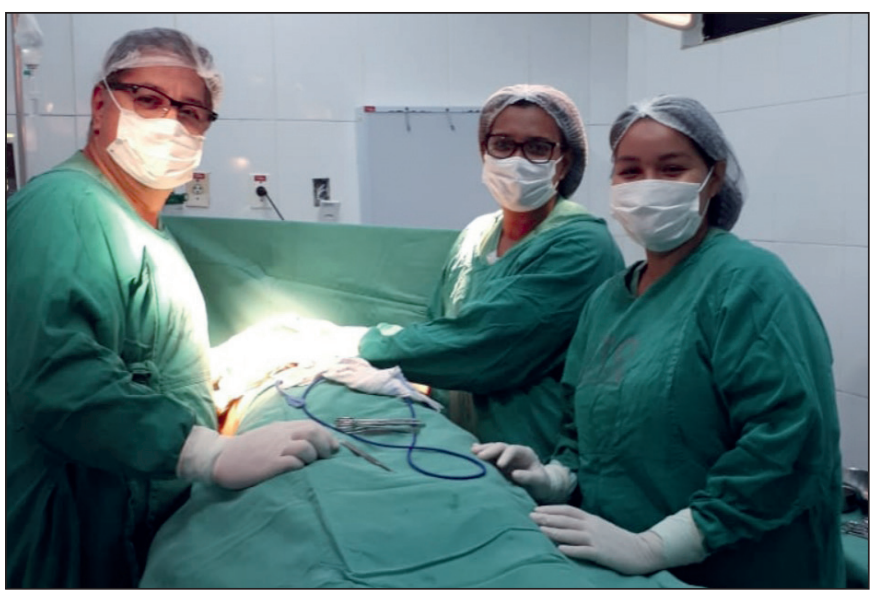

Figura 8. Equipo del Dr.Cesar Kelly Villafuerte, Brasil.

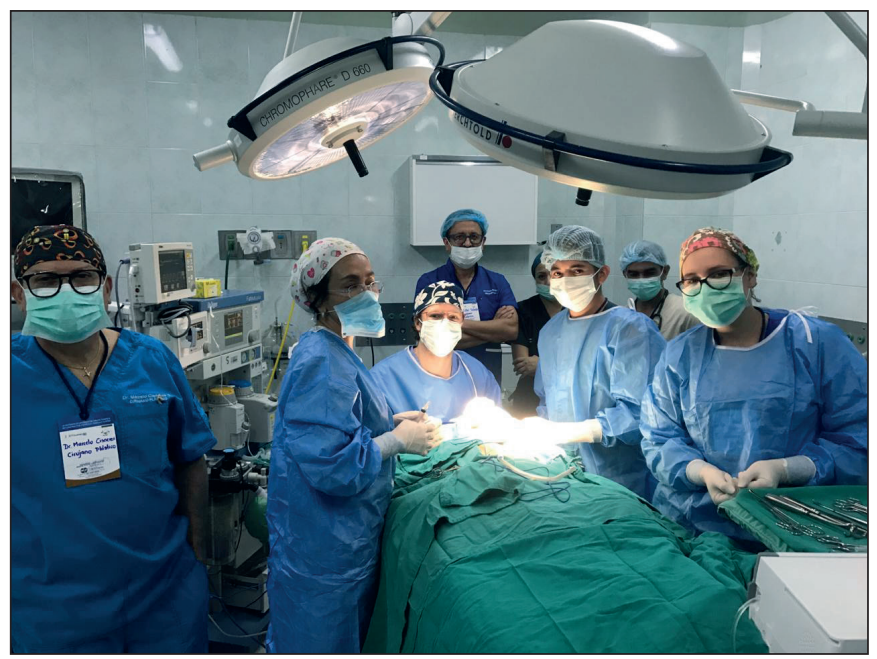

Figura 10. Equipo del Dr. Edison Ramos, Ecuador.

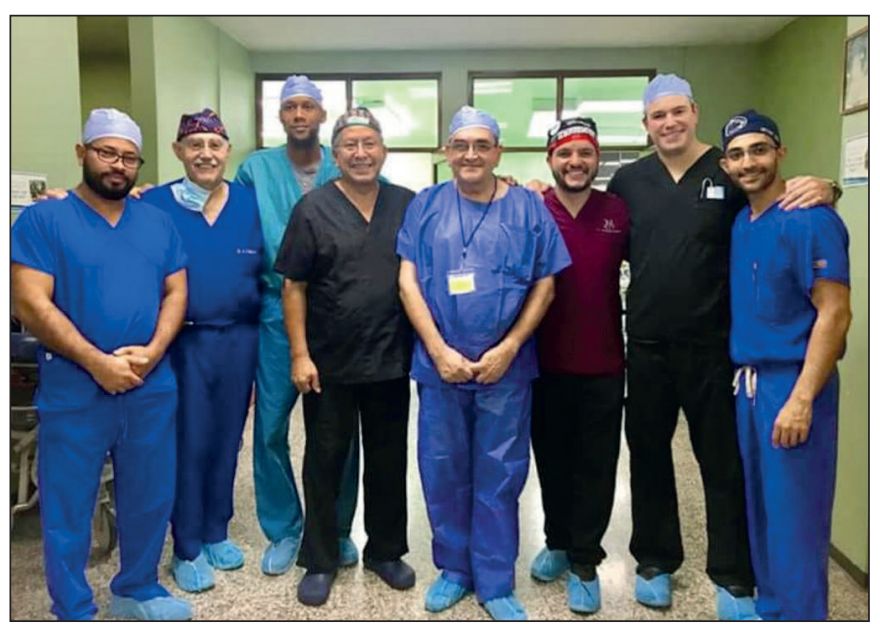

Figura 12. Equipo del Dr. Carlos Vacaflor, Bolivia.

las que muchas veces son excluidos del grupo social o mantenidos ocultos o alejados de los demás, pueden acceder a una mejora en sus vidas y a su reinserción social y/o laboral. ${ }^{(3-6)}$

En ocasiones, simples injertos, colgajos cutáneos locales y Z-plastias pueden resolver o mejorar casos muy incapacitantes o deformantes de retracciones o secuelas de quemaduras en pacientes que, por escasez de medios económicos o de transporte, en áreas geográficas de difí- 


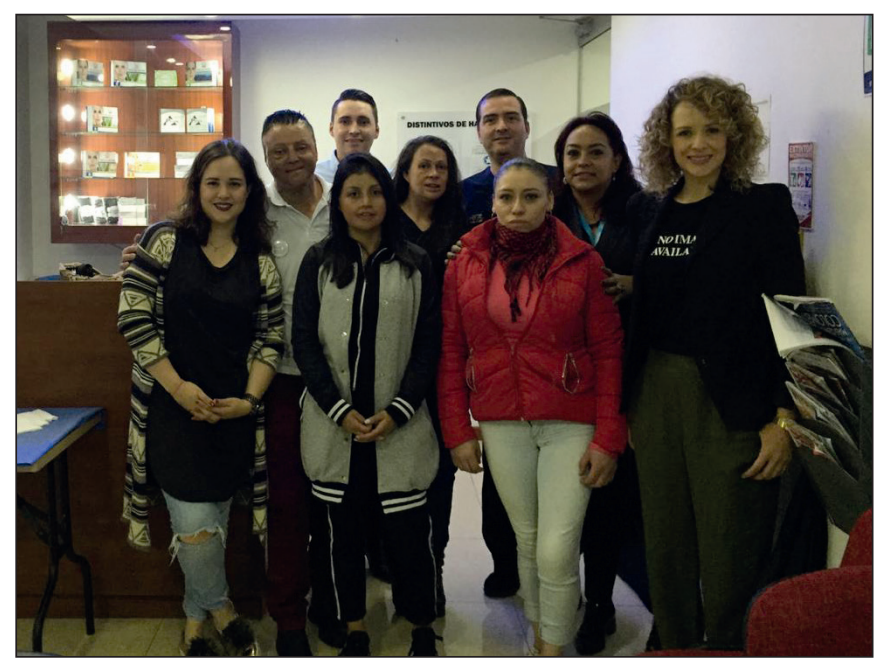

Figura 13. Equipo del Dr. Marco Antonio Salazar, Colombia.

cil acceso o alejadas de los grandes centros hospitalarios en los que se prestan servicios especializados, se ven relegados socialmente y privados de hacer una vida normal o desarrollar una actividad laboral con la que ganarse la vida. ${ }^{(7)}$ En este tipo de campaña, esta es una actividad sencilla cuando se componen grupos de trabajo centralizados que convocan a numerosos pacientes que pueden ser operados con anestesia local, en régimen ambulatorio, sin necesidad de grandes cuidados postoperatorios y con un rápido retorno a su localidad de origen. Una fácil solución cuando la brindan manos especializadas y en equipos bien organizados. ${ }^{(8)}$

Lo mismo podemos decir en otras situaciones más complejas, como es el caso de los niños afectados por labio o paladar hendido en los que una cirugía especializada, en la edad y momento oportunos, puede brindarles excelentes beneficios tanto para su alimentación y desarrollo normales como para su integración social, escolarización y desarrollo como personas. ${ }^{(9)}$ Aparecen en ocasiones también en este tipo de campañas adultos con secuelas de esta patología no resueltas en su momento o con intervenciones que no se llegaron a completar, y que también pueden beneficiarse de un cambio importante en sus vidas. Nos resulta especialmente interesante el dato de que si bien el rango de edad de los pacientes tratados en esta III Campaña de FILACP osciló entre los 3 meses y los 76 años, la media de edad fue de 9.6 años, es decir, con un marcado enfoque en la población infantil.

El tercer pilar de la presente campaña fue la reconstrucción mamaria postcáncer, un aspecto de nuestra labor quirúrgica al que cada vez más mujeres acceden en todos los países del ámbito Latinoamericano, pero que muchas veces no está totalmente cubierto por la sanidad nacional en costos o en infraestructuras y en el que la labor altruista de nuestros cirujanos se desarrolla últimamente con gran interés. Sobre este tema, se han publicado ya también algunos trabajos en esta misma revista. ${ }^{(10,11)}$
Las Jornadas Altruistas FILACP se iniciaron con la filosofía inculcada por los maestros de ayudar a quienes menos tienen. Se crearon durante la presidencia del Dr. Rómulo Guerrero con una jornada de trabajo convocada para el 26 de mayo de 2009 y continuaron durante la presidencia del Dr. Guillermo Vázquez con la supervisión del mismo Dr. Guerrero, en aquel momento Director de Relaciones Internacionales de la FILACP, en la campaña del 22 de julio del año 2016. Observando las grandes necesidades de atención a la salud que existen en nuestros países, es durante la gestión del Dr. Alejandro Duarte, actual Presidente de la FILACP, cuando se decide ampliar las fechas a dos meses de actividad, para así tratar de proporcionar un mayor plazo de cobertura que permitiera la participación de más grupos quirúrgicos y en más países. Somos conscientes de que una campaña de 2 meses, en la que cada equipo puede trabajar una media de 5-6 días, no va a cambiar la desigualdad social en la que viven los pacientes atendidos ni sus condiciones básicas de vida; pero sin embargo, sí creemos que resulta de gran ayuda si tenemos en cuenta que nuestra cirugía, y solo hecha por nuestros especialistas, es precisamente la que requieren estos pacientes para mejorar su situación social y su salud. De otra manera, en la mayoría de los casos, no tendrían posibilidad alguna de opción quirúrgica para sus dolencias.

A través del presente artículo, La FILACP agradece a todos y cada uno de los cirujanos que fueron parte de esta III Jornada Altruista y a la vez reconoce a todos los que participaron en las campañas anteriores por el trabajo sincero, dedicado y silencioso de cada uno de ellos. Pretende también que sirva de modelo y guía para futuras campañas, y que con su lectura, los más jóvenes especialistas se animen a integrarse en la actividad.

Como coordinadores de la presente campaña, pero también como participantes activos en la misma, podemos afirmarles con certeza que además todos los que participaron lo hicieron con voluntad y compromiso desinteresados, y que gracias al trabajo realizado por todos, la FILACP, en poco tiempo, se convertirá en una organización reconocida a nivel mundial por la labor social y altruista que realiza a través de los cirujanos de sus 22 países miembros.

\section{Conclusiones}

Hacemos del conocimiento de la sociedad en general la labor altruista de los cirujanos plásticos de la FILACP con la descripción, a modo de ejemplo de la labor realizada en los últimos años, de esta III Campaña Altruista internacional convocada en el año 2019.

Pretendemos también contribuir al cambio de la principal imagen que damos los cirujanos plásticos a nivel 
mundial, demostrando que no solo la vertiente estética de la especialidad enfoca nuestra labor, sino que es mucho lo que podemos hacer para mejorar la calidad de vida y la integración social de los pacientes aquejados por malformaciones congénitas, secuelas de quemaduras, accidentes o cáncer.

\section{Dirección del autor}

Dra. Teresa Zambrana Rojas

Av. Ayacucho 0174, $2^{\circ}$, ofic. 6

Edificio María Antonieta

Cochabamba, Bolivia

Correo electrónico: tezamr@hotmail.com

Bibliografía

1.- Historia de la FILACP www.filacp.org

2.- Labor social de la Cirugía Plástica Ibero Latinoamericana www.ciplaslatin.com

3.- Vallarta Rodríguez A, Morales Olivera JM, Duarte y Sánchez A. La Cirugía Plástica y su labor humanitaria en México. Cir. Plást. iberolatinoam 2015; 41(3): 335-343.
4.- Thomas Bas C., Fonfach Zahn C. Trabajo voluntario para disminución de listas de espera de Cirugía Plástica en hospitales públicos de Chile. Operativo Hospital de Valdivia, enero 2019. Cir. Plást. iberolatinoam 2019; 45(2): 203-205.

5.- Sigler A. Protocolo para la planificación quirúrgica en las clínicas de labio y paladar hendidos en la zona noroeste de la República Mexicana. Cir. plást. iberolatinoam. 2017; 43(3):313-325

6.- Novoa Rodríguez M., López Suso E., et al. Labor humanitaria en África. Nuestra experiencia. Cir plást iberolatinoam 2019; 45(3):327-338.

7.- Laredo Ortíz C., García Bernal F., et al. Nuestra experiencia en campaña humanitaria en Liberia. Cir plást iberolatinoam 2018; 44(2):231-242.

8.- Barón Thaidigsmann J. Campañas quirúrgicas humanitarias de la Sociedad Española de Cirugía Plástica, Reparadora y Estética en colaboración con los Hermanos de San Juan de Dios. Cir plást iberolatinoam 2017; 43(4):425-437.

9.- De la Cruz Acosta, F. Operación Sonrisa República Dominicana: 8 años de una intensa y positiva experiencia. Cir plást iberolatinoam 2016; 42(1):93-101.

10.- Arroyo F, Ortega L. Campaña de reconstrucción mamaria "Mujeres apoyando mujeres". Cir. plást. iberolatinoam. 2017; 43(1): 97-102.

11.- Jiménez Muñoz-Ledo G., De León Jasso A., et al. Campañas de reconstrucción mamaria en Guanajuato, México. Cir. plást. iberolatinoam. 2018; 44(4): 449-453. 
